\title{
Soft drop thrust in lepton collisions
}

\author{
Zoltán Trócsányi*t \\ Institute for Theoretical Physics, ELTE Eötvös Loránd University, \\ Pázmány Péter sétány 1/A, H-1117 Budapest, Hungary \\ and MTA-DE Particle Physics Research Group, H-4010 Debrecen, PO Box 105, Hungary \\ E-mail: Zoltan. Trocsanyi@cern.ch
}

We discuss the status of the strong coupling with special attention to using event shape observables based on data collected at the Large Electron Positron collider and theoretical predictions at highest accuracy available at present. We argue that such extractions can be competitive with lattice results if we select the event shapes sufficiently carefully such that both non-perturbative and higher order perturbative contributions are as small as possible. We give a list of such observables and study one particular class in detail. We present predictions for the soft drop thrust at the next-to-next-to-leading order (NNLO) accuracy in QCD perturbation theory and study the scale dependence as a function of the grooming parameters.

European Physical Society Conference on High Energy Physics - EPS-HEP2019 -

10-17 July, 2019

Ghent, Belgium

\footnotetext{
* Speaker.

${ }^{\dagger}$ Work supported by grant K 125105 of the National Research, Development and Innovation Fund in Hungary.
} 


\section{Introduction}

According to the recent summary on the status of the strong coupling [1], the current world average value, $\alpha_{\mathrm{s}}\left(M_{Z}\right)=0.1181 \pm 0.0011$, is dominated by the lattice results [2] that show the smallest uncertainties by far, somewhat below $1 \%$. The results of the experimental measurements span a much larger range, over $4 \%$. The largest spread of $\alpha_{\mathrm{s}}\left(M_{Z}\right)$ values appears among the extractions from distributions of the geometrical properties of hadronic final states in electronpositron annihilation, which is somewhat against the expectations because such collisions provide a clean environment with strong interactions affecting only the final state. The main reasons for the large uncertainties lie in the usually large perturbative and non-perturbative (hadronization) effects. One can expect improved results only by including higher-order corrections. During the last 15 years, i.e. after the closure of LEP, we witnessed significant advances in improving the perturbative description of the event shape distributions. On the one hand the next-to-next-to-leading order (NNLO) corrections have been computed for three-jet like observables [3, 4, 5], while on the other resummation of large logarithms to all orders have been performed at the next-to-next-to-leading logarithmic (NNLL or $\left.\mathrm{N}^{2} \mathrm{LL}\right)$ ) and in some cases even at $\mathrm{N}^{3} \mathrm{LL}$ accuracy $[6,7,8]$. Both resulted in a significant decrease of the dependence on the unphysical renormalization scale, hence on the theoretical uncertainty.

\section{Event shapes at $\mathbf{N N L O}+\mathrm{N}^{3} \mathrm{LL}+\mathrm{PC}$}

We can see the impressive improvement of the theoretical predictions with the increasing order in the truncations of the perturbation series on the example of the thrust $(\tau=1-T)$ distribution $[9,10]$. In Fig. 1 (left) we see the predictions at the first three orders in perturbation theory (at LO, NLO and NNLO accuracy), as given by the perturbative expansion for the normalized cross section,

$$
\frac{\tau}{\sigma} \frac{\mathrm{d} \sigma}{\mathrm{d} \tau}=\left(\frac{\alpha_{\mathrm{s}}}{2 \pi}\right) A(\tau)+\left(\frac{\alpha_{\mathrm{s}}}{2 \pi}\right)^{2} B(\tau)+\left(\frac{\alpha_{\mathrm{s}}}{2 \pi}\right)^{3} C(\tau) .
$$

We have computed the $A, B$ and $C$ coefficients using the MCCSM program [12] based on the CoLoRFuINNLO subtraction method $[13,14]$. One can also see that even the predictions at NNLO are significantly smaller than the data over the whole kinematic range, but especially for small values of $\tau$ where the $\operatorname{logarithms} L=-\ln \tau$ become large. The latter feature is readily understood from the analytic structure of perturbative predictions:

$$
\begin{aligned}
& A(\tau)=A_{1} L+A_{0}, \\
& B(\tau)=B_{3} L^{3}+B_{2} L^{2}+B_{1} L+B_{0}, \\
& C(\tau)=C_{5} L^{5}+C_{4} L^{4}+C_{3} L^{3}+C_{2} L^{2}+C_{1} L+C_{0}
\end{aligned}
$$

where the dependence of the coefficients on $\tau$ is suppressed. In order to obtain a reliable prediction for small values of $\tau$, the logarithmic terms have to be resummed to all orders in perturbation theory. Fig. 1 (left) shows the combined NNLO and $\mathrm{N}^{3}$ LL predictions. The same logarithms appear in both predictions at low orders, and we used R-matching [15] to avoid the double counting of those terms. The matched prediction exhibits improved agreement between theory and data for the whole thrust distribution as compared to that at NNLO. Nevertheless, there still remains a large gap between the 
two in the peak region where most of the data fall. We assume that the main source of difference between the perturbative prediction and the data is hadronization.
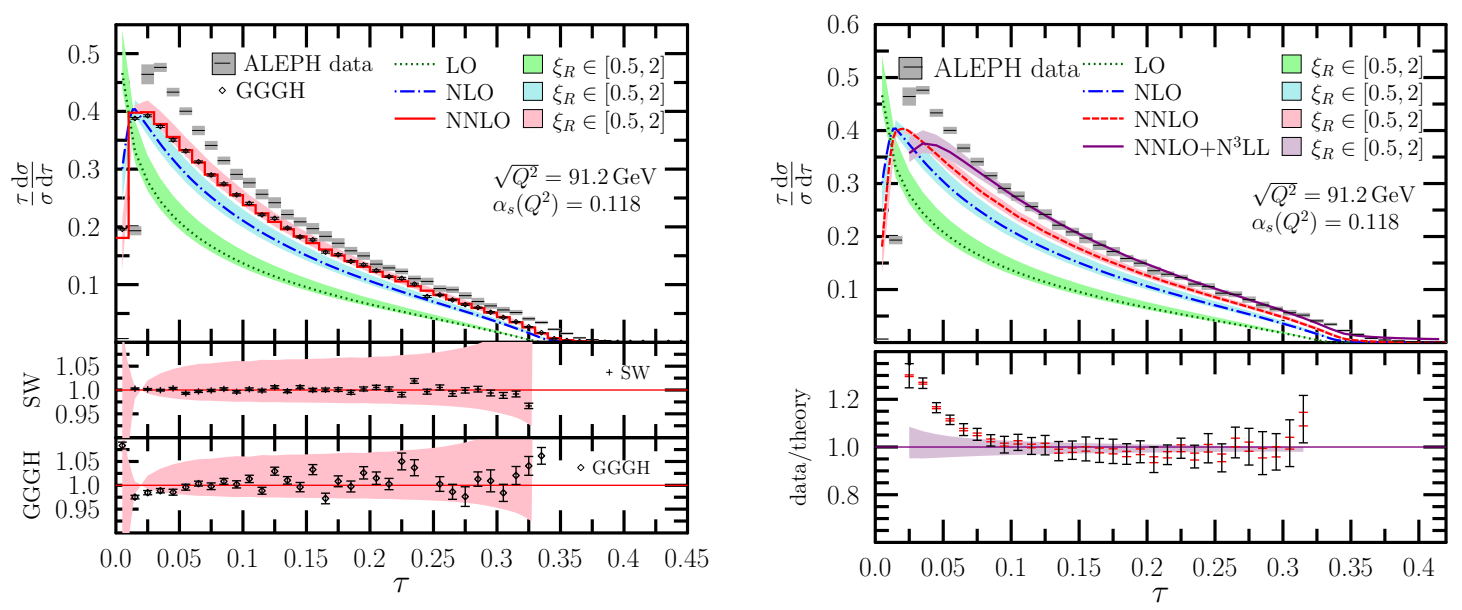

Figure 1: Distributions of thrust in electron-positron annihilation normalized to the total hadornic cross section $\sigma$. Left: predictions at first three orders in perturbation theory and also matched NNLO and $\mathrm{N}^{3} \mathrm{LL}$ predictions obtained with the world average for the strong coupling. Right: distribution of matched NNLO and $\mathrm{N}^{3} \mathrm{LL}$ predictions supplemented with analytic model for power corrections fitted to ALEPH data. The bands represent the variation of the renormalization scale $\mu_{R}=\xi_{R} \sqrt{Q^{2}}$ around the default one $\left(\xi_{R}=1\right)$ where $\sqrt{Q^{2}}$ is the total centre-of-mass energy. The range $\xi_{R} \in[0.5,2]$ is the accepted measure of the uncertainty in the perturbative predictions at fixed order in perturbation theory. The lower panels show the ratio of the (updated but unpublished) predictions of Ref. [4] (SW) and the EERAD3 code [11] (GGGH) to CoLoRFUINNLO.

There are two options for estimating the hadronization corrections: (i) there are analytic models (power corrections, or PC) for them [6, 16, 17], or (ii) one can simulate hadronization effects using modern Monte Carlo programs. Both methods have their caveats. The analytic model contains one or two non-perturbative parameters that have to be fitted simultaneously with the strong coupling. One expects that both the perturbative and the non-perturbative parameters have to be independent of the event shape variable. We see on Fig. 2 that this expectation about universality is not fulfilled by the fits of the predictions at NNLO+N $\mathrm{N}^{3} \mathrm{LL}$ accuracy supplemented with power corrections. The fits also show very strong anti-correlation between the perturbative and nonperturbative parameters [18].

The estimation of hadronization effects by MC tools uses simulations of the final states both at the parton and at the hadron level. Estimating the distributions from these simulations, one takes the ratio of the two predictions as a multiplicative correction factor (fitted with a smooth function) $[19,20]$. Hence the correction factor is model dependent. We can decrease the effect of this model dependence on the extracted value of the strong coupling if we select shape variables with small hadronozation corrections ("large uncertainty in small quantity is small uncertainty"). Such an observable is the jet cone energy fraction [21], for which the NNLO corrections are very small except near the edges of the phase space [5]. 

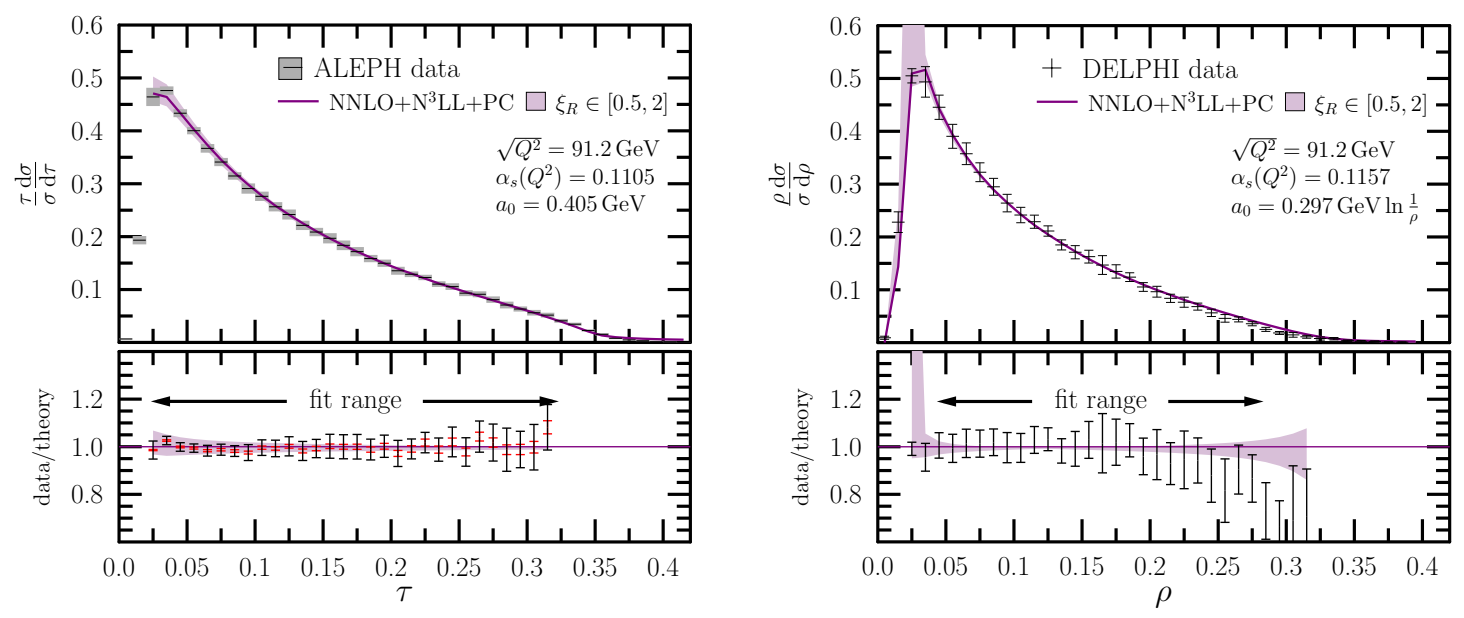

Figure 2: Distributions of thrust ( $\tau$, left) and heavy jet mass ( $\rho$, right) in electron-positron annihilation, normalized to the total hadronic cross section $\sigma$ and using matched NNLO and $\mathrm{N}^{3}$ LL predictions supplemented with power corrections for hadronization fitted to Delphi data. The bands represent the variation of the renormalization scale around the default one as in Fig. 1.

\section{Soft drop event shapes}

The soft drop grooming was introduced in Ref. [22], further developed in Ref. [23], and defined for jets produced in lepton collisions in Ref. [24]. It was designed to decrease the effect of soft gluons on the distributions. As the soft gluons have a major role in the hadronization, one expects that the groomed event shapes exhibit smaller hadronization corrections. Indeed, it was shown in Ref. [25] that the soft-drop thrust distribution suffers smaller hadronization corrections that its ungroomed counterpart. We studied [26] the perturbative behaviour of the version $T_{\mathrm{SD}}^{\prime}$ of the soft-drop thrust that is free of a transition point in the soft-collinear region Ref. [25]. This event shape variable depends on two grooming parameters $\beta$ and $z_{\text {cut }}$. The effect of these parameters on hadronization corrections was studied It was shown in Ref. [25] that with stronger grooming, i.e. decreasing $\beta$ and increasing $z_{\text {cut }}$, the hadronization corrections to the distribution of $T_{\mathrm{SD}}^{\prime}$ decrease over a wide range of the event shape. Unfortunately, at the same time the cross section also decreases, and the optimal values of the grooming parameters have to be found by taking into account other effects that might influence the quality of the agreement between theory and data.

Such effect is the rate of convergence of the perturbation series, which is characterized by the NLO and NNLO $K$-factors defined by ratios of distributions as

$$
K_{\mathrm{NLO}}(\mu)=\frac{\mathrm{d} \sigma_{\mathrm{NLO}}(\mu)}{\mathrm{d} T_{\mathrm{SD}}^{\prime}} / \frac{\mathrm{d} \sigma_{\mathrm{LO}}(Q)}{\mathrm{d} T_{\mathrm{SD}}^{\prime}}, \quad K_{\mathrm{NNLO}}(\mu)=\frac{\mathrm{d} \sigma_{\mathrm{NNLO}}(\mu)}{\mathrm{d} T_{\mathrm{SD}}^{\prime}} / \frac{\mathrm{d} \sigma_{\mathrm{LO}}(Q)}{\mathrm{d} T_{\mathrm{SD}}^{\prime}}
$$

In order to see the convergence of the perturbation expansion more directly, we also define the ratio of the NNLO predictions to NLO ones, denoted by

$$
K_{\mathrm{NNLO}^{\prime}}(\mu)=\frac{\mathrm{d} \sigma_{\mathrm{NNLO}}(\mu)}{\mathrm{d} T_{\mathrm{SD}}^{\prime}} / \frac{\mathrm{d} \sigma_{\mathrm{NLO}}(Q)}{\mathrm{d} T_{\mathrm{SD}}^{\prime}}
$$



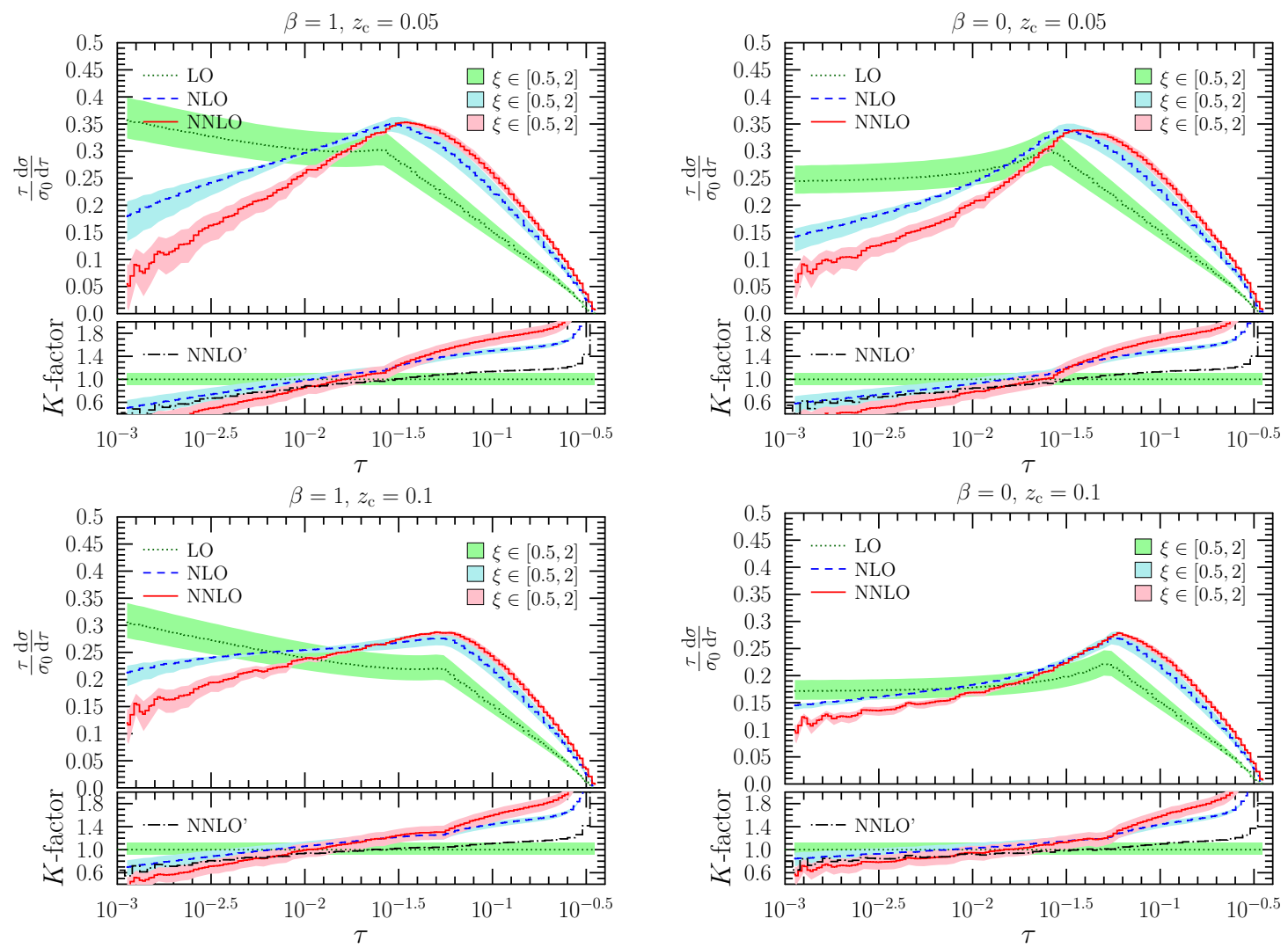

Figure 3: Distributions of the soft drop thrust $\tau=1-T_{\mathrm{SD}}^{\prime}$ at LO, NLO and NNLO accuracy for various values of the grooming parameters as indicated above each plot. The lower sections exhibit the $K$-factors (the $\mathrm{LO} K$-factor, $K_{\mathrm{LO}}=1$ is shown for reference). The bands represent the variation of the renormalization scale around the default one in the range $\left[\mu_{0} / 2,2 \mu_{0}\right]$. In the case of $K_{\mathrm{NNLO}^{\prime}}$ the scale dependence does not provide relevant information, hence not shown.

In these equations we computed the cross sections in the denominators at the default renormalization scale $\mu=Q$, independently of $\mu$. The closer the $K$-factors to unity, the better the convergence of the perturbative series. In order to check how grooming affects the perturbative convergence, we scanned the region of $\left(\beta, z_{\text {cut }}\right)$ values over the rectangle spanned by the corners $\{(0,0.05),(1,0.05),(0,0.1),(1,0.1)\}$. We found that the $K$-factors depend on the grooming parameters smoothly. Fig. 3 shows the distribution and the $K$-factors at the corners of this rectangle. We see that stronger the grooming results in better perturbative convergence. The values $\beta=0$ and $z_{\text {cut }}=0.1$ look optimal in the sense that the cross section still remains sizeable. The same conclusions can be drawn if one uses the soft drop hemisphere mass [26].

\section{Conclusions}

In this talk we discussed that precise determination of the strong coupling using hadronic final states in electron-positron annihilation requires (i) careful selection of observables, (ii) estimation of the hadronization corrections with modern MC tools and (iii) needs methods to reduce hadronization corrections. The latter could be some grooming techniques, such as soft drop. We 
used the MCCSM program for computing differential distributions for the soft-drop thrust (and also hemisphere mass, but not shown here) at the NNLO accuracy. We observed that soft drop improves the perturbative convergence of the predictions. The smaller perturbative uncertainty, together with the reduced hadronization corrections makes the soft-drop thrust and hemisphere jet mass appealing candidates for a precise determination of the strong coupling at lepton colliders. Indeed, in Ref. [27] the extraction of $\alpha_{\mathrm{s}}\left(M_{Z}\right)$ was performed using matched NLO+NLL prediction for the soft drop thrust. The result is promising, and the reduction of the hadronization uncertainty is observed. To make such extractions even more precise, it will be important to take into account the effect of resummation of large logarithmic terms, which is in progress for the case of hemisphere jet mass.

\section{References}

[1] D. d'Enterria, $\alpha_{s}$ status and perspectives (2018), in 26th International Workshop on Deep Inelastic Scattering and Related Subjects (DIS 2018) Port Island, Kobe, Japan, April 16-20, 2018, 2018. 1806.06156.

[2] ALPHA Collaboration, M. Bruno, M. Dalla Brida, P. Fritzsch, T. Korzec, A. Ramos, S. Schaefer, H. Simma, S. Sint and R. Sommer, QCD Coupling from a Nonperturbative Determination of the Three-Flavor $\Lambda$ Parameter, Phys. Rev. Lett. 119 (2017), no. 10102001 [1706.03821].

[3] A. Gehrmann-De Ridder, T. Gehrmann, E. W. N. Glover and G. Heinrich, NNLO corrections to event shapes in e+e- annihilation, JHEP 12 (2007) 094 [0711.4711].

[4] S. Weinzierl, Event shapes and jet rates in electron-positron annihilation at NNLO, JHEP 06 (2009) $041[0904.1077]$.

[5] V. Del Duca, C. Duhr, A. Kardos, G. Somogyi, Z. Szőr, Z. Trócsányi and Z. Tulipánt, Jet production in the CoLoRFulNNLO method: event shapes in electron-positron collisions, Phys. Rev. D94 (2016), no. 7074019 [1606.03453].

[6] T. Becher and M. D. Schwartz, A precise determination of $\alpha_{s}$ from LEP thrust data using effective field theory, JHEP 07 (2008) 034 [0803.0342].

[7] R. Abbate, M. Fickinger, A. H. Hoang, V. Mateu and I. W. Stewart, Thrust at $N^{3} L L$ with Power Corrections and a Precision Global Fit for alphas(mZ), Phys. Rev. D83 (2011) 074021 [1006.3080].

[8] A. H. Hoang, D. W. Kolodrubetz, V. Mateu and I. W. Stewart, C-parameter distribution at $N^{3} L L \hat{a} \breve{A} \breve{s}$ including power corrections, Phys. Rev. D91 (2015), no. 9094017 [1411.6633].

[9] S. Brandt, C. Peyrou, R. Sosnowski and A. Wroblewski, The Principal axis of jets. An Attempt to analyze high-energy collisions as two-body processes, Phys. Lett. 12 (1964) 57-61.

[10] E. Farhi, A QCD Test for Jets, Phys. Rev. Lett. 39 (1977) 1587-1588.

[11] A. Gehrmann-De Ridder, T. Gehrmann, E. Glover, and G. Heinrich, "EERAD3: Event shapes and jet rates in electron-positron annihilation at order $\alpha_{s}^{3}$," Comput.Phys.Commun. 185 (2014) 3331, arXiv:1402.4140 [hep-ph].

[12] A. Kardos, G. Somogyi and Z. Trócsányi, Jet cross sections with CoLoRFul NNLO, PoS LL2016 (2016) 021.

[13] G. Somogyi, Z. Trocsanyi and V. Del Duca, A Subtraction scheme for computing QCD jet cross sections at NNLO: Regularization of doubly-real emissions, JHEP 01 (2007) 070

[hep-ph/0609042]. 
[14] G. Somogyi and Z. Trocsanyi, A Subtraction scheme for computing QCD jet cross sections at NNLO: Regularization of real-virtual emission, JHEP 01 (2007) 052 [hep-ph / 060904 3].

[15] S. Catani, Y. L. Dokshitzer, M. Olsson, G. Turnock and B. R. Webber, New clustering algorithm for multi - jet cross-sections in e+e-annihilation, Phys. Lett. B269 (1991) 432-438.

[16] Y. L. Dokshitzer and B. R. Webber, Calculation of power corrections to hadronic event shapes, Phys. Lett. B 352, 451 (1995) [hep-ph/9504219].

[17] T. Gehrmann, G. Luisoni and P. F. Monni, Power corrections in the dispersive model for a determination of the strong coupling constant from the thrust distribution, Eur. Phys. J. C73 (2013), no. 12265 [1210.6945].

[18] Y.-T. Chien and M. D. Schwartz, Resummation of heavy jet mass and comparison to LEP data, JHEP 08 (2010) 058 [1 005.1644$].$

[19] A. Kardos, S. Kluth, G. Somogyi, Z. Tulipánt and A. Verbytskyi, Precise determination of $\alpha_{S}\left(M_{Z}\right)$ from a global fit of energyâĂŞenergy correlation to NNLO+NNLL predictions, Eur. Phys. J. C78 (2018), no. 6498 [1804.09146].

[20] A. Verbytskyi, A. Banfi, A. Kardos, P. F. Monni, S. Kluth, G. Somogyi, Z. Szőr, Z. Trócsányi, Z. Tulipánt and G. Zanderighi, High precision determination of $\alpha_{s}$ from a global fit of jet rates, Submitted to: JHEP (2019) [1902.08158].

[21] Y. Ohnishi and H. Masuda, The Jet cone energy fraction in e+ e- annihilation, SLAC-PUB-6560.

[22] M. Dasgupta, A. Fregoso, S. Marzani and G. P. Salam, Towards an understanding of jet substructure, JHEP 09 (2013) 029 [1307.0007].

[23] A. J. Larkoski, S. Marzani, G. Soyez and J. Thaler, Soft Drop, JHEP 05 (2014) 146 [1 402 . 2657 ].

[24] C. Frye, A. J. Larkoski, M. D. Schwartz and K. Yan, Factorization for groomed jet substructure beyond the next-to-leading logarithm, JHEP 07 (2016) 064 [1603. 09338].

[25] J. Baron, S. Marzani and V. Theeuwes, Soft-Drop Thrust, JHEP 08 (2018) 105 [1803. 04719 ].

[26] A. Kardos, G. Somogyi and Z. Trócsányi, Soft-drop event shapes in electron-positron annihilation at next-to-next-to-leading order accuracy, Phys. Lett. B786 (2018) 313-318 [1807 . 11472].

[27] S. Marzani, D. Reichelt, S. Schumann, G. Soyez and V. Theeuwes, Fitting the Strong Coupling Constant with Soft-Drop Thrust, arXiv:1906.10504 [hep-ph]. 